\title{
Using electric fields to prevent mirror-trapped antiprotons in antihydrogen studies
}

\author{
P. Richerme, ${ }^{1}$ G. Gabrielse, ${ }^{1,}{ }^{*}$ S. Ettenauer, ${ }^{1}$ R. Kalra, ${ }^{1}$ E. Tardiff, ${ }^{1}$ D. W. Fitzakerley, ${ }^{2}$ M. C. George, ${ }^{2}$ E. A. Hessels, ${ }^{2}$ \\ C. H. Storry, ${ }^{2}$ M. Weel, ${ }^{2}$ A. Müllers, ${ }^{3}$ and J. Walz ${ }^{3}$ \\ (ATRAP Collaboration) \\ ${ }^{1}$ Department of Physics, Harvard University, Cambridge, Massachusetts 02138, USA \\ ${ }^{2}$ York University, Department of Physics and Astronomy, Toronto, Ontario, Canada M3J 1P3 \\ ${ }^{3}$ Institut für Physik, Johannes Gutenberg Universität and Helmholtz Institut Mainz, D-55099 Mainz, Germany
}

(Received 24 August 2012; published 25 February 2013)

\begin{abstract}
The signature of trapped antihydrogen $(\overline{\mathrm{H}})$ atoms is the annihilation signal detected when the magnetic trap that confines the atoms is suddenly switched off. This signal would be difficult to distinguish from the annihilation signal of any trapped $\bar{p}$ that is released when the magnetic trap is switched off. This work deduces the large cyclotron energy $(>137 \mathrm{eV})$ required for magnetic trapping of $\bar{p}$, considers the possibility that such $\bar{p}$ are produced, and explores the effectiveness of an electric field applied to clear charged particles from the trapping volume before $\overline{\mathrm{H}}$ detection. No mechanisms are found that can give a $\bar{p}$ such a large cyclotron energy and allow it to mimic an $\overline{\mathrm{H}}$ annihilation. The method used to release $\overline{\mathrm{H}}$ atoms from their magnetic trap without removing the magnetic field gradient that could possibly confine $\bar{p}$ with a high cyclotron energy is also discussed.
\end{abstract}

DOI: 10.1103/PhysRevA.87.023422

PACS number(s): 37.10.De, 36.10.-k, 41.20.-q, 41.90.+e

\section{INTRODUCTION}

Antihydrogen atoms have recently been trapped for up to about $1000 \mathrm{~s}$ by both the ATRAP and ALPHA Collaborations at CERN [1,2]. ATRAP observed an average of 5 atoms per trial confined for $\geqslant 15 \mathrm{~s}$, while ALPHA trapped approximately $0.7 \overline{\mathrm{H}}$ atoms per trial for $\geqslant 10 \mathrm{~s}$ using smaller numbers of antiprotons $(\bar{p})$ and positrons $\left(e^{+}\right)$. These are important steps towards the proposed use of trapped $\overline{\mathrm{H}}$ atoms [3] for $C P T$ tests with precision spectroscopy [4] and for proposed gravitational studies with trapped antimatter atoms [5], though larger numbers of trapped atoms will be required.

The $\overline{\mathrm{H}}$ atoms are confined within the magnetic field minimum of a Ioffe trap [6] to which the magnetic moment of weak-field seeking states of the neutral $\overline{\mathrm{H}}$ atoms are attracted. The trapped $\overline{\mathrm{H}}$ atoms are detected via their annihilations with the surrounding apparatus when the current producing the radial Ioffe fields is switched off. It is important that the signal used to identify and count an $\overline{\mathrm{H}}$ atom is from a trapped atom and not from a trapped $\bar{p}$ that is released at the same time, given that an $\overline{\mathrm{H}}$ and a $\bar{p}$ have the same annihilation signals.

A $\bar{p}$ that is given a large cyclotron energy may have a magnetic moment that is large enough to be captured magnetically. The simplest "mirror trap" in which a $\bar{p}$ with cyclotron energy could be confined is a magnetic field that increases in magnitude to either side of the trap center-just the situation that pertains when the axially symmetric contribution to a Ioffe trap field is turned on. A charged $\bar{p}$ is fixed to the field line around which it has its circular cyclotron orbit, and the magnetic moment is confined axially by the gradient magnetic field. Already some time ago, particles in a mirror trap [7] were used to measure the electron and positron magnetic

\footnotetext{
*gabrielse@ physics.harvard.edu
}

Published by the American Physical Society under the terms of the Creative Commons Attribution 3.0 License. Further distribution of this work must maintain attribution to the author(s) and the published article's title, journal citation, and DOI. moments [8], to directly trap $e^{+}$for multispecies plasma experiments [9], and to bunch $e^{+}[10]$.

This paper discusses the $\bar{p}$ cyclotron energy needed to mirror trap a $\bar{p}$ in the ATRAP Ioffe trap, considers mechanisms for producing these large energies, and examines the effectiveness of the electric field that is applied to clear away possible mirrortrapped $\bar{p}$ while the $\overline{\mathrm{H}}$ atoms are trapped, before these atoms are detected. We seek (unsuccessfully) to identify processes that can provide a $\bar{p}$ with the cyclotron energy necessary for it to remain mirror-trapped when the clearing field is applied. As an added precaution, the $\overline{\mathrm{H}}$ atoms are released for detection while the mirror trap from the axially symmetric Ioffe field coils is left on. Mirror trapping requires considerably more $\bar{p}$ cyclotron energy in our apparatus compared to another that has been studied [11].

\section{TRAPPED $\overline{\mathrm{H}}$ ATOMS}

The apparatus used to observe trapped $\overline{\mathrm{H}}$ atoms in their ground state is represented in Fig. 1. The hollow copper cylinders can be biased as Penning trap electrodes to make open-access Penning traps [12] for $\bar{p}$ and $e^{+}$. Currents in the Ioffe pinch coils and the Ioffe racetrack coils make a magnetic field minimum that is centered on these coils. The potentials on the electrodes are manipulated such that $e^{+}$and $\bar{p}$ interact to form $\overline{\mathrm{H}}$ atoms at the field minimum.

$\overline{\mathrm{H}}$ formation at this location proceeds via three-body recombination involving a $\bar{p}$ and two $e^{+}[13,14]$ within a nested-well potential [Fig. 2(a)]. Virtually all of the atoms formed are "guiding center atoms" [14] that cannot be magnetically trapped. Some of these atoms are observed to form in states with $n \lesssim 50$ [15]. This atom size is just small enough that the $e^{+}$orbit about the $\bar{p}$ is chaotic, opening the possibility of occasional low-field-seeking states that can be trapped [15]. Some of these atoms remain in low-field-seeking states during their cascade to the ground state [1,2], consistent with simulations $[16,17]$.

To be confined an $\overline{\mathrm{H}}$ atom must have a very low kinetic energy. The trap potential energy contours in Fig. 2(b) are for 


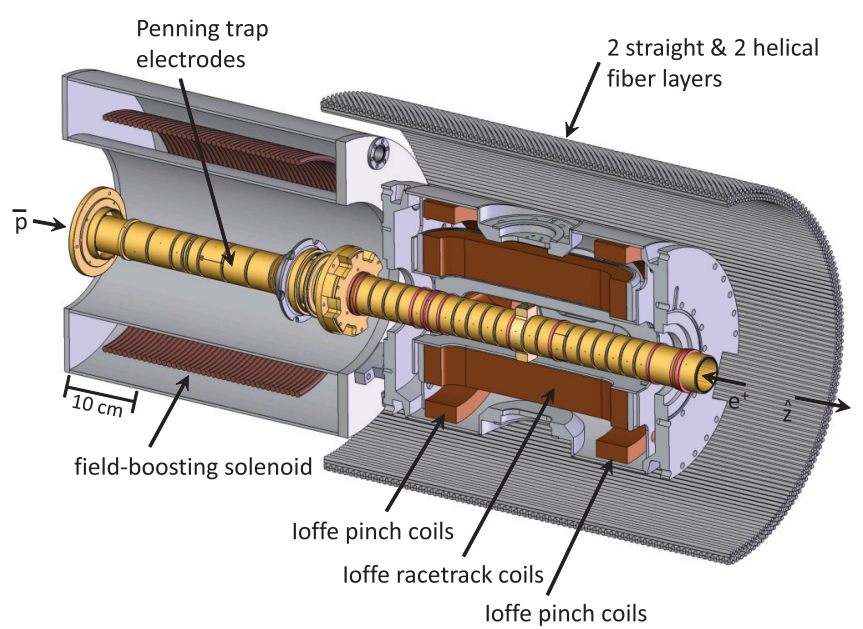

FIG. 1. (Color) Electrodes and coils produce Penning traps (to store $\bar{p}$ and $e^{+}$) and a Ioffe trap [6] (to store $\overline{\mathrm{H}}$ ). Much of the vacuum enclosure and cooling system is hidden to make the traps and detectors visible. An external solenoid (not shown) adds a $1 \mathrm{~T}$ magnetic field along the trap axis $\hat{z}$ which is vertical.

a ground-state $\overline{\mathrm{H}}$. The magnetic field on the center axis of the trap is shown in Fig. 2(c).

ATRAP holds trapped $\overline{\mathrm{H}}$ atoms in the Ioffe trap for between 15 and $1000 \mathrm{~s}$ (depending upon the method used to make $\bar{p}$ and $e^{+}$interact to form $\overline{\mathrm{H}}$ )-long enough for $\overline{\mathrm{H}}$ atoms to decay to their ground state [1]. While the $\overline{\mathrm{H}}$ are trapped, axial electric fields of first $+5 \mathrm{~V} / \mathrm{cm}$ and then $-5 \mathrm{~V} / \mathrm{cm}$ are applied [e.g., the dashed line in Fig. 2(a)], each for $1 \mathrm{~s}$, to force all $\bar{p}$ and $e^{+}$
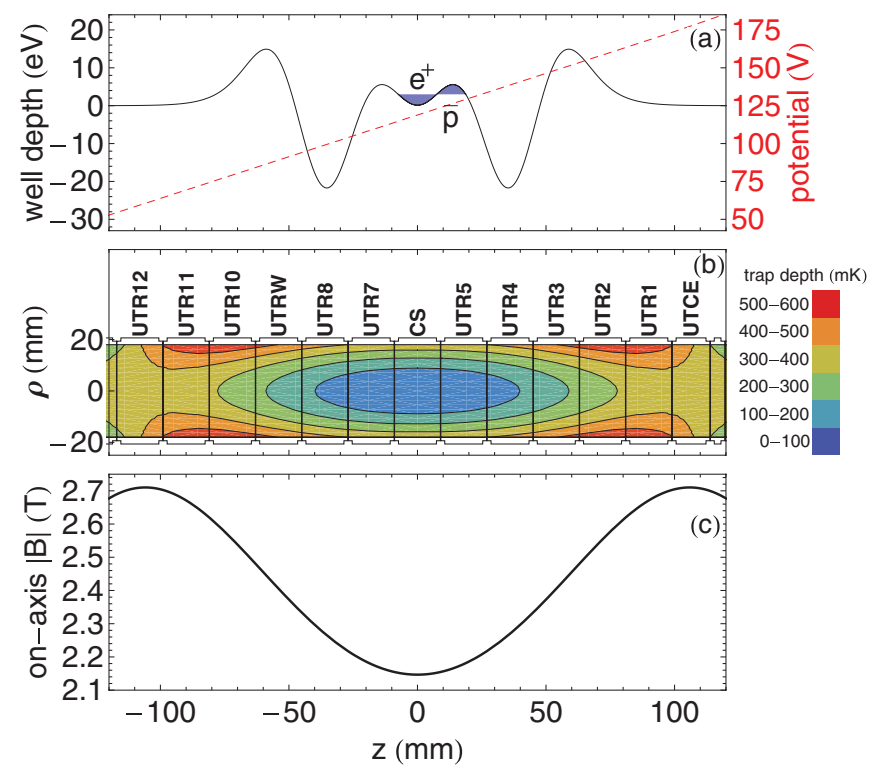

FIG. 2. (Color) (a) Potentials along the center axis of the trap used to contain charged $\bar{p}$ and $e^{+}$until $\overline{\mathrm{H}}$ formation (solid) and remove (dashed) charged particles after $\overline{\mathrm{H}}$ formation. (b) Trap electrode cross sections with equipotential energy contours for a low-field-seeking, ground-state $\overline{\mathrm{H}}$ in the Ioffe trap. (c) The magnitude of the on-axis magnetic field rises from $2.15 \mathrm{~T}$ at the center of the trap to $2.71 \mathrm{~T}$ near the pinch coils. to leave the trap. These clearing electric fields are much larger than any stray fields within the Penning trap.

Trapped $\overline{\mathrm{H}}$ atoms leave the trap radially when the current in the Ioffe racetrack coils that produces the radial Ioffe field is switched off. The annihilation signals the $\overline{\mathrm{H}}$ atoms make upon striking electrode surfaces [1] are detected.

\section{CYCLOTRON ENERGY NEEDED FOR MIRROR TRAPPING}

During $\overline{\mathrm{H}}$ detection, the mirror trap from the Ioffe pinch coils is left on to maximize the chance that a mirror-trapped $\bar{p}$ (if any are present) remains confined. However, possible transient effects upon such $\bar{p}$ from the sudden removal of the radial Ioffe field are not well understood. Thus, it would be better to be confident that no $\bar{p}$ at all were stored in the mirror trap. As a first step this section considers the cyclotron energy needed to produce a magnetic moment large enough to remain trapped when the clearing electric field is applied.

A $\bar{p}$ in the combined fields of a Penning and Ioffe trap executes a modified cyclotron, axial, and magnetron motion compared to its motion in a simple Penning trap [18]. Adiabatic invariants [19] have been identified for each of these motions, provided that the magnetic field changes sufficiently slowly along the $\bar{p}$ trajectory so that orbit frequencies change by only a small fraction during an orbit period, a condition easily satisfied here. For a $\bar{p}$ with cyclotron energy $E_{c}$, its magnetic moment $\mu_{\bar{p}}=E_{c} /|\vec{B}|$ is an adiabatic invariant of the cyclotron motion. As $|\vec{B}|$ increases, $E_{c}$ also increases to keep $\mu_{\bar{p}}$ fixed.

The sum of the $\bar{p}$ cyclotron energy $E_{c}$ and the $\bar{p}$ translational kinetic energy $E_{T}$ also remains fixed. Thus as a $\bar{p}$ moves from a region of weak to strong $|\vec{B}|, E_{c}$ increases to keep $\mu_{\bar{p}}$ invariant, and $E_{T}$ decreases to keep the total energy constant. If the $\bar{p}$ moves into a $|\vec{B}|$ large enough to reduce the translational energy to $E_{T}=0$, the $\bar{p}$ reverses its trajectory and in this sense is "mirror-trapped."

Consider a $\bar{p}$ with kinetic energy $E_{T}$ and cyclotron energy $E_{c}$ in a magnetic field, $B_{0}$. If the $\bar{p}$ moves to a larger magnetic field $B$ it will not be mirror-trapped if its initial kinetic energy is high enough,

$$
E_{T}>E_{c}\left(\frac{B}{B_{0}}-1\right) \text {. }
$$

The center of the ATRAP Ioffe trap has $B_{0}=2.15 \mathrm{~T}$ and $B_{\max }=2.71 \mathrm{~T}\left[\right.$ Fig. 2(c)]. Thus a kinetic energy $E_{T}>0.26 E_{c}$ at the trap center ensures that a $\bar{p}$ will not be mirror-trapped. In a $\bar{p}$ plasma with cyclotron and translational motions thermalized at $4 \mathrm{~K}$, an unacceptably large fraction of $8 \%$ would be mirror-trapped.

For this reason the strong clearing electric field is introduced to sweep away $\bar{p}$ after $\overline{\mathrm{H}}$ formation. The applied force must be stronger than both the stray fields within the trap electrodes and the mirror-trap restoring force. When a potential $\Phi$ is applied, an additional term, $-e \Phi$, must be added to the total energy (where $-e$ is the $\bar{p}$ charge). Equating the total energy at the center of the trap, where $|\vec{B}|=B_{0}$ and $\Phi=\Phi_{0}$, to the energy at some other point parametrized by $B$ and $\Phi$, mirror-trapping 
is avoided if

$$
E_{T}>E_{c}\left(\frac{B}{B_{0}}-1\right)-e\left(\Phi-\Phi_{0}\right) .
$$

For large enough $\Phi-\Phi_{0}$ it is possible to make sure that no $\bar{p}$ is mirror-trapped [i.e., to satisfy Eq. (2)] for any $E_{c}$ and $B$, even when $E_{T}=0$.

For ATRAP, only a $\bar{p}$ with an extremely high cyclotron energy can remain mirror-trapped after the clearing electric field is applied. Clearing fields of both $+5 \mathrm{~V} / \mathrm{cm}$ and $-5 \mathrm{~V} / \mathrm{cm}$ are used [e.g., $+5 \mathrm{~V} / \mathrm{cm}$ is the dashed line in Fig. 2(a)] and $\left(B_{\max } / B_{0}-1\right)=0.26$. This means that a minimum cyclotron energy $E_{c}>137 \mathrm{eV}$ is required for a $\bar{p}$ with no translational kinetic energy ( $E_{c}>200 \mathrm{eV}$ in the center of the Ioffe trap) to be mirror-trapped. This limit is rather conservative given that a $\bar{p}$ that has no translational energy along a magnetic field line and a high cyclotron energy is very unlikely. Note that since $\left(B_{\max } / B_{0}-1\right)=0.26$ in ATRAP, compared with $\left(B_{\max } / B_{0}-\right.$ $1)=1.0$ in the ALPHA apparatus that has been studied [11], we may tolerate a markedly larger value of $E_{c}(137 \mathrm{eV}$ vs. $50 \mathrm{eV}$ ) while still satisfying Eq. (2) (for the same $E_{T}$ and $\Phi$ ).

Above we have considered only the case of a $\bar{p}$ with no translational energy at the center of the Ioffe trap. Mirror trapping is much less likely in general. To determine whether a $\bar{p}$ with a given $E_{c}$ at other locations will remain mirror-trapped with the clearing potential applied, we consider first the most restrictive case $E_{T}=0$. The right-hand side of Eq. (2) can then be treated as a pseudopotential,

$$
V=-e\left(\Phi-\Phi_{0}\right)+E_{c}\left(\frac{B}{B_{0}}-1\right),
$$

through which the $\bar{p}$ moves. A $\bar{p}$ remains mirror-trapped only if it stays in a local minimum of the pseudopotential.

The easiest case to visualize is that of a $\bar{p}$ that is on the central axis of the trap since it will stay on this axis. The on-axis potential $\Phi(z)$ is the dashed line in Fig. 2(a), and the on-axis magnetic field strength $B(z)$ is shown in Fig. 2(c). Figure 3(a) shows the on-axis pseudopotential energy as a function of axial position for three values of the cyclotron energy $E_{c}$. With a $5 \mathrm{~V} / \mathrm{cm}$ electric clearing field applied, the pseudopotential contains no local minimum until $E_{c}>137 \mathrm{eV}$. Thus, any $\bar{p}$ on-axis with $E_{c}<137 \mathrm{eV}$ will be swept away by the clearing potential. Those with $E_{c}>137 \mathrm{eV}$ can remain mirror-trapped but only if they remain spatially localized in the pseudopotential well with too small an energy $E_{T}$ to escape.

If a $\bar{p}$ on-axis starts with $E_{T}=0$ at a higher potential energy far to the left in Fig. 3(a), it may still gain enough kinetic energy to escape over the barrier to the right even if $E_{c}>137 \mathrm{eV}$. Figure 3(b) plots the minimum cyclotron energy a $\bar{p}$ would need, along with $E_{T}=0$, to remain mirror-trapped when starting from different values of $z$ on-axis. The curve reaches a minimum at $137 \mathrm{eV}$. Away from the minimum, the curve rises to the left on account of the longer distance over which the clearing potential can accelerate $\bar{p}$. The curve rises to the right as $\bar{p}$ are no longer localized in the confining well of the pseudopotential. Note that for $\bar{p}$ located at the center of the nested well $(z=0)$, only those with $E_{c}>200 \mathrm{eV}$ could potentially survive the electric clearing field.

The off-axis trajectories for a $\bar{p}$ in a Penning-Ioffe trap are more complicated due to the presence of the radial Ioffe

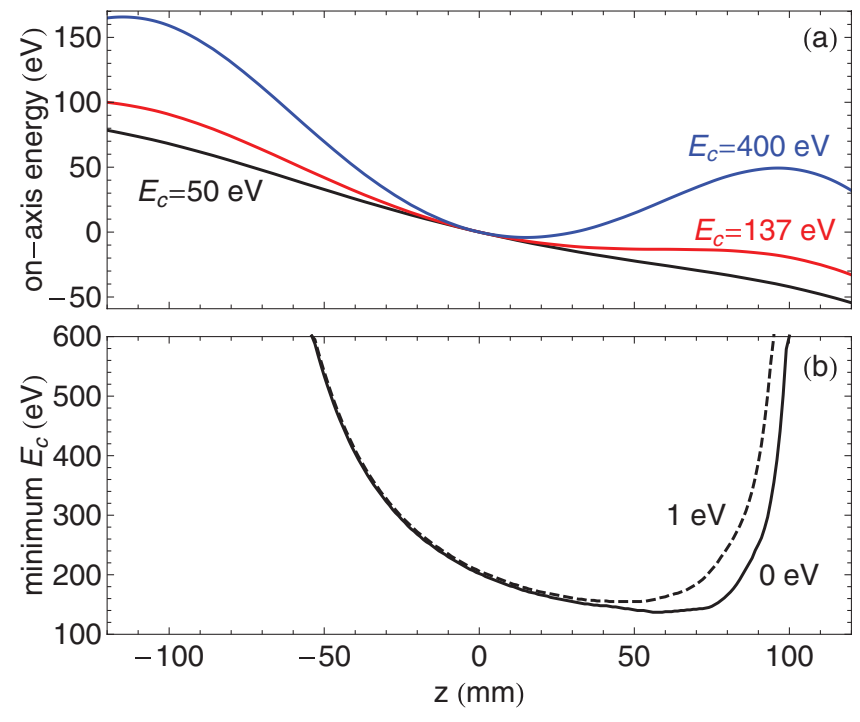

FIG. 3. (Color online) (a) The pseudopotential on-axis is plotted for three different values of $\bar{p}$ cyclotron energy $E_{c}$ when an electric clearing field of $5 \mathrm{~V} / \mathrm{cm}$ is applied. At $E_{c}=137 \mathrm{eV}$, the pseudopotential just begins to show a local minimum. (b) The minimum value $E_{c}$ a $\bar{p}$ would need to remain mirror-trapped when starting at different axial positions $z$ for $E_{T}=0$ and $E_{T}=1 \mathrm{eV}$ and the corresponding pseudopotential in Eq. (3). More cyclotron energy is needed for mirror trapping in some locations for the larger $E_{T}$.

field. The magnetic field lines, some of which are shown in Fig. 4(a), are no longer along $\hat{z}$. They diverge exponentially in the planes aligned with the Ioffe current bars (the long vertical sections of the racetrack coils shown in Fig. 1). Nonetheless, the pseudopotential along these field lines may be determined using Eq. (3) with calculated off-axis potentials and the calculated off-axis magnetic field, as illustrated in Fig. 4(b).

To determine if any $\bar{p}$ with $E_{c}<137 \mathrm{eV}$ may be mirrortrapped off-axis, we calculate trajectories for $\bar{p}$ with various $E_{c}$ and $E_{T}=0$ at a grid of starting locations in the trap. To speed

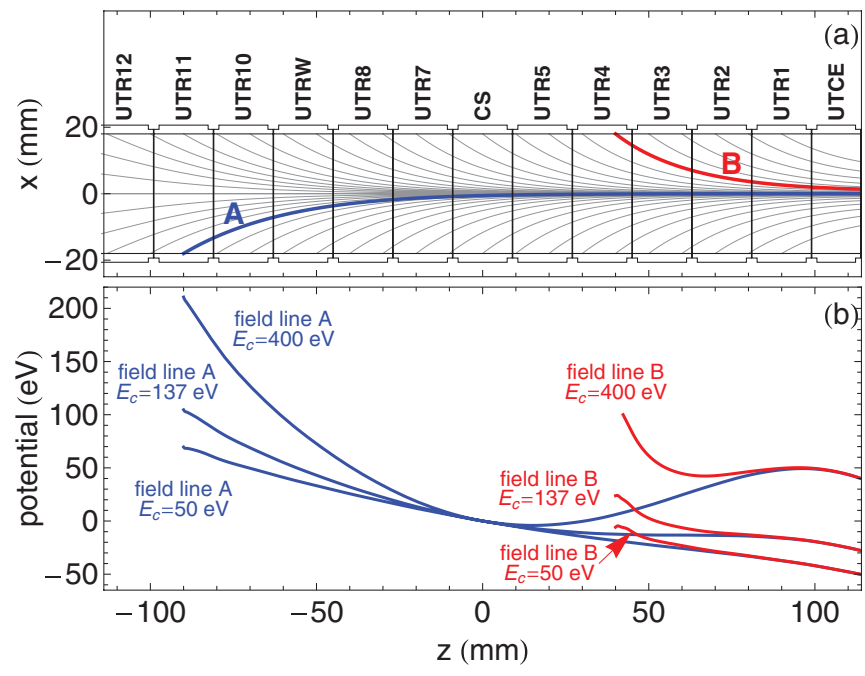

FIG. 4. (Color) (a) A sample of the maximally divergent magnetic field lines in the Penning-Ioffe trap. For various values of $E_{c}$, the pseudopotential along the two indicated field lines is plotted in panel (b), projected on the $z$ axis. 


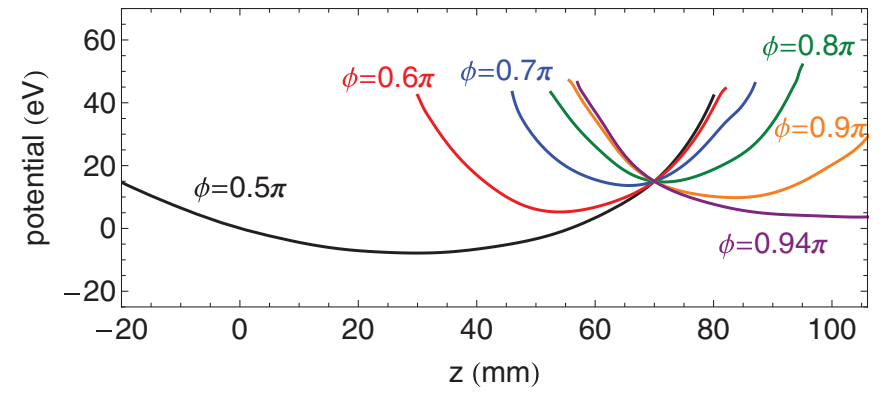

FIG. 5. (Color) The value of the pseudopotential is plotted along magnetic field lines passing through $\rho=10 \mathrm{~mm}$, for various $\phi$ and $z=70 \mathrm{~mm}$, for a $\bar{p}$ with $E_{c}=200 \mathrm{eV}$. Though the $\bar{p}$ may be temporarily mirror-trapped when $\phi=0.5 \pi$, magnetron drift allows the $\bar{p}$ to escape once its azimuthal angle crosses $\phi=0.94 \pi$.

calculations, we employ the guiding-center approximation, in which the fast cyclotron motion is replaced by the adiabatically invariant magnetic moment $\mu_{\bar{p}}$ that remains aligned with the local magnetic field [20]. Trajectories for $\bar{p}$ off-axis are thus excursions along a magnetic field line with a slight change in azimuthal angle during each bounce due to the magnetron drift.

A $\bar{p}$ will only remain mirror-trapped if it stays in a local minimum of the pseudopotential throughout its entire trajectory. For example, consider a $\bar{p}$ with $E_{c}=200 \mathrm{eV}$ starting at the coordinates $\rho=10 \mathrm{~mm}, \phi=\pi / 2$, and $z=70 \mathrm{~mm}$. This $\bar{p}$ will be mirror-trapped initially, as shown in Fig. 5. However, as the $\bar{p}$ magnetron drift changes the azimuthal angle $\phi$, the pseudopotential becomes less confining until, at $\phi=0.94 \pi$, the $\bar{p}$ escapes. A $\bar{p}$ is confined in a stable mirror-trapped trajectory if the pseudopotential is confining for all $\phi$. Otherwise, the trajectory intersects a trap electrode or exits out the end of the trap.

Figure 6 shows the result of the trajectory calculations. For each of the initial grid locations the minimum initial value of $E_{c}$ that results in mirror trapping is represented. The lowest cyclotron energy that results in mirror trapping is $E_{c}=137 \mathrm{eV}$ on the trap axis [Fig. 3(b)]. A $\bar{p}$ at any location in the trap will not be mirror-trapped if its initial cyclotron energy is less than $137 \mathrm{eV}$.

\section{ACQUIRING MORE THAN $137 \mathrm{eV}$ OF CYCLOTRON ENERGY}

To minimize the chance that mirror-trapped $\bar{p}$ would be released and counted as $\overline{\mathrm{H}}$ atoms, we do not turn off the axial

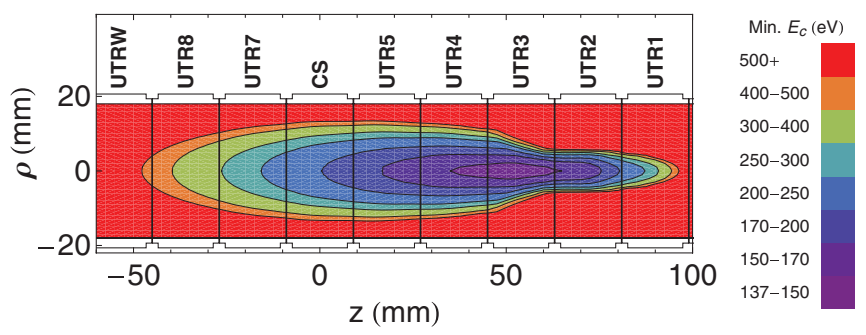

FIG. 6. (Color) The cyclotron energy $E_{c}$ that a $\bar{p}$ must have at each location in the trap if it is to be mirror-trapped as determined by trajectory calculations. The global minimum, on the center axis of the trap, is $E_{c}=137 \mathrm{eV}$, so no $\bar{p}$ with $E_{c}<137 \mathrm{eV}$ is mirror-trapped. mirror trap (from the Ioffe pinch coils of Fig. 1) when releasing trapped $\overline{\mathrm{H}}$ atoms to count them. Instead, only the currents in the Ioffe racetracks (Fig. 1) are switched off. It would be safer, of course, if no $\bar{p}$ was mirror-trapped when the racetracks are turned off. There would then be no possibility that turn-off transients not well understood could eject a trapped $\bar{p}$, for example.

This section considers whether any $\bar{p}$ could acquire enough cyclotron energy to be mirror-trapped at the time that $\overline{\mathrm{H}}$ are released for detection. The previous section concluded that to be mirror-trapped a $\bar{p}$ would need to acquire at least $137 \mathrm{eV}$ of cyclotron energy (more than $10^{6} \mathrm{~K}$ in temperature units) for a $\bar{p}$ located on-axis between electrodes UTR3 and UTR4 (Fig. 6). For most $\bar{p}$ locations, and for $E_{T}>0$, even more than this high cyclotron energy would be required.

Evaluating the cyclotron energy that a $\bar{p}$ would have to have at the time at which $\overline{\mathrm{H}}$ atoms are released from the trap requires a brief consideration of some aspects of the capture and cooling of $\bar{p}$. These familiar processes have been described elsewhere [21]. Possible thermal and nonthermal excitations of $\bar{p}$ cyclotron motion are considered, along with the ionization of $\overline{\mathrm{H}}$ atoms.

\section{A. Cooled and uncooled $\bar{p}$}

The $\bar{p}$ for these measurements are initially captured by ATRAP in a 5-keV potential well, whereupon they can have translational energies along $3.7 \mathrm{~T}$ magnetic field lines up to the well depth. Insofar as some trapped $\bar{p}$ could have cyclotron energies that are similarly large they would be candidates for mirror trapping.

Most of the initially captured $\bar{p}$ collide with cold trapped electrons [21]. This electron cooling thermalizes both the $\bar{p}$ cyclotron energy and the $\bar{p}$ translational energy along magnetic field lines. As discussed in the following section, the thermalized cyclotron energy is much too small for mirror trapping.

Any $\bar{p}$ that is radially outside the cold electron plasma does not collide with the electrons and is thus not electron-cooled. Uncooled $\bar{p}$ with large cyclotron energies are actually ejected from the trap volume not long after the $\bar{p}$ are initially captured in the ATRAP Penning trap (long before the Ioffe racetrack current is turned off to let trapped $\overline{\mathrm{H}}$ atoms escape). The loss takes place when the magnetic field $B_{i}=3.7 \mathrm{~T}$ in which the $\bar{p}$ are initially captured is adiabatically reduced to $B_{f}=1 \mathrm{~T}$. The ratio of the cyclotron energy and the magnetic field is an adiabatic invariant, as discussed earlier. As a result the $\bar{p}$ cyclotron energy reduces from $E_{c i}$ to $E_{c f}=E_{c i}\left(B_{f} / B_{i}\right)$. The lost cyclotron energy increases the translational energy along the magnetic field lines by $\Delta E=E_{c f}\left(B_{i} / B_{f}-1\right)=2.7 E_{c f}$. Thus $E_{c f}=137 \mathrm{eV}$ is only possible for a translational energy greater than $370 \mathrm{eV}$.

After the magnetic field reduction, the well depth for the Penning trap is only $10 \mathrm{eV}$, ensuring that $\bar{p}$ with enough cyclotron energy for later mirror trapping are ejected from the trap. A $\bar{p}$ with more than about $4 \mathrm{eV}$ of cyclotron energy would have acquired enough energy to escape the $10-\mathrm{eV}$ well.

When the Ioffe trap is turned on the magnetic field increases to $2.1 \mathrm{~T}$. The cyclotron energy increases in proportion but would still be very much less than what is needed for mirror trapping. 


\section{B. Thermal $\bar{p}$}

After catching, cooling, and transferring $\bar{p}$ to a nested Penning trap, the $\bar{p}$ temperature before $\overline{\mathrm{H}}$ formation has been directly measured to be $31 \mathrm{~K}$ (as part of a study of subsequent adiabatic cooling [22]). For a $\bar{p}$ density of $10^{6} \mathrm{~cm}^{-3}$, the isotropization rate between axial and cyclotron energy is $330 \mathrm{~Hz}$ at our magnetic field [23], which is fast enough to ensure that the axial and cyclotron temperatures remain in thermal equilibrium with each other. For a thermal distribution of cyclotron energies centered around $E_{c}=31 \mathrm{~K}=2.7 \mathrm{meV}$, the probability of having $E_{c}>137 \mathrm{eV}$ is negligibly small.

\section{Driven $\bar{p}$}

Forming $\overline{\mathrm{H}}$ requires increasing the amplitude of the motion of the $\bar{p}$ along the magnetic field lines to bring them into contact with the $e^{+}$plasma. To this end, radio-frequency electric fields are applied to drive this $\bar{p}$ motion. To keep the drives near resonance with the $\bar{p}$ oscillation along the magnetic field lines, the drive frequency is either chirped or broadened by noise. The $\bar{p}$ cyclotron energy is not directly excited, since applied oscillating electric fields are far off resonance and are cylindrically symmetric. Instead, the $\bar{p}$ gain translational energy along magnetic field lines, and collisions equilibrate the translational and cyclotron motions, leaving them both in a thermal distribution at the same temperature.

For every set of drive parameters used, the axial $\bar{p}$ temperature has been measured following their excitation. In the most extreme case, when the strongest noise-broadened drive was applied for $10 \mathrm{~min}$, the $\bar{p}$ axial (and hence cyclotron) energy was found to be $1 \mathrm{eV}$. For a thermal distribution at this energy, for example, only a single $\bar{p}$ out of $10^{6}$ has a cyclotron energy larger than $15 \mathrm{eV}$. Since all $\bar{p}$ with $E_{c}<$ $137 \mathrm{eV}$ are ejected by the clearing electric field, the probability that a single $\bar{p}$ could remain mirror-trapped is negligible.

Upper bounds on the translational energy can also be set by observing $\bar{p}$ loss rates. Approximately 500 out of $10^{6} \bar{p}$ in a $3-\mathrm{eV}$ thermal distribution, for example, would have enough translational energy to escape the 27-eV-deep nested well. Since such loss is not observed, the $\bar{p}$ energy must always be less than $3 \mathrm{eV}$ (consistent with our direct measurements). Even more stringent bounds may be placed by observing the number of $\bar{p}$ escaping over the 5-eV central nested well barrier, with the result that in all cases the probability of creating a mirror-trapped $\bar{p}$ during the drive is negligible.

As the $\bar{p}$ mix with $e^{+}$to form $\overline{\mathrm{H}}$, they are likely to lose cyclotron energy since the two species equilibrate at a rate faster than the recombination rate. The $e^{+}$cooling of $\bar{p}$ has long been demonstrated [24], with a collisional cooling rate of approximately $100 \mathrm{~s}^{-1}$ for an $e^{+}$plasma with a density of $5 \times 10^{7} \mathrm{~cm}^{-3}$ in a 2.2-T magnetic field [25]. At the $31 \mathrm{~K} e^{+}$ temperature, the three-body recombination rate [13] is nearly 2 orders of magnitude smaller than the collisional cooling rate. This affords sufficient time for any $\bar{p}$ with large amounts of cyclotron energy to lose it in collisions with the $e^{+}$.

\section{Ionized $\overline{\mathbf{H}}$}

Most of the $\overline{\mathrm{H}}$ atoms produced are in highly excited states that can be ionized with a laboratory electric field. A $\bar{p}$ from an ionized $\overline{\mathrm{H}}$ atom could have most of the kinetic energy of the $\overline{\mathrm{H}}$, along with any energy acquired by acceleration of the $\bar{p}$ after ionization.

The energy distribution of the $\bar{p}$ produced by the ionization of an $\overline{\mathrm{H}}$ atom is predominantly the energy distribution of the $\bar{p}$ from the $\overline{\mathrm{H}}$ formed. The reasons that $\bar{p}$ have much less than $137 \mathrm{eV}$ of cyclotron energy have already been discussed.

A $\bar{p}$ from $\overline{\mathrm{H}}$ ionization oscillates along a magnetic field line with an energy that arises from the electrical potential where the ionization takes place, possibly increased slightly by the small kinetic energy from the $\overline{\mathrm{H}}$ motion just considered. At most, this conveys $40 \mathrm{eV}$ of energy (if the ionization takes place just inside the electrode wall and if the $\bar{p}$ avoids striking an electrode surface). Collisions with $e^{+}$or other $\bar{p}$ are much too rare to convert this oscillation energy into cyclotron energy and to boost the cyclotron energy above $137 \mathrm{eV}$.

No plausible way that $\overline{\mathrm{H}}$ ionization can produce the minimum $137 \mathrm{eV}$ of $\bar{p}$ cyclotron energy that is required for mirror trapping has thus been identified.

\section{CONCLUSION}

In conclusion, the signals that establish that an average of five $\overline{\mathrm{H}}$ atoms per trial have been confined in a quadrupole Ioffe trap for 15 to $1000 \mathrm{~s}$ seem to be properly attributed to $\overline{\mathrm{H}}$ atoms rather than to mirror-trapped $\bar{p}$ that are released at the same time. The mirror-trap field is not turned off when $\overline{\mathrm{H}}$ atoms are released to make it less likely that mirror-trapped $\bar{p}$, if some did exist, would be released with the trapped $\bar{H}$. In addition, this study shows that a minimum of $137 \mathrm{eV}$ of cyclotron energy (more than $10^{6} \mathrm{~K}$ in temperature units) must be given to a $\bar{p}$ for it to be mirror-trapped in ATRAP's Ioffe trap. Much more cyclotron energy is required at most $\bar{p}$ locations in the Penning-Ioffe trap and for $\bar{p}$ with translational energy. No plausible source of such high cyclotron energies has been identified.

\section{ACKNOWLEDGMENTS}

We are grateful to CERN for delivering the $5-\mathrm{MeV} \bar{p}$ from its unique Antiproton Decelerator. This work was supported by the NSF and the AFOSR of the United States; the NSERC, the CRC, the CFI, and the OIT of Canada; and the BMBF and the DFG of Germany.
[1] G. Gabrielse, R. Kalra, W. S. Kolthammer, R. McConnell, P. Richerme, D. Grzonka, W. Oelert, T. Sefzick, M. Zielinski, D. W. Fitzakerley, M. C. George, E. A. Hessels, C. H. Storry, M. Weel, A. Müllers, and J. Walz, Phys. Rev. Lett. 108, 113002 (2012).
[2] G. B. Andresen et al. (ALPHA Collaboration), Nat. Phys. 7, 558 (2011)

[3] G. Gabrielse, in Fundamental Symmetries, edited by P. Bloch, P. Pavlopoulos, and R. Klapisch (Plenum, New York, 1987), pp. 59-75. 
[4] C. Zimmermann and T. W. Hänsch, Hyperfine Interact. 76, 47 (1993).

[5] G. Gabrielse, Hyperfine Interact. 44, 349 (1988).

[6] D. E. Pritchard, Phys. Rev. Lett. 51, 1336 (1983).

[7] R. C. Davidson, Physics of Non-neutral Plasmas (Imperial College Press, London, 2001).

[8] J. C. Wesley and A. Rich, Phys. Rev. A 4, 1341 (1971).

[9] H. Boehmer, M. Adams, and N. Rynn, Phys. Plasmas 2, 4369 (1995).

[10] J. A. P. Mills, Appl. Phys. 22, 273 (1980).

[11] C. Amole et al. (ALPHA Collaboration), New J. Phys. 14, 015010 (2012).

[12] G. Gabrielse, L. Haarsma, and S. L. Rolston, Int. J. Mass Spectrom. Ion Processes 88, 319 (1989); 93, 121 (1989).

[13] G. Gabrielse, S. L. Rolston, L. Haarsma, and W. Kells, Phys. Lett. A 129, 38 (1988).

[14] M. Glinsky and T. O’Neil, Phys. Fluids B 3, 1279 (1991).

[15] G. Gabrielse, Adv. At. Mol. Opt. Phys. 50, 155 (2005).
[16] T. Pohl, H. R. Sadeghpour, Y. Nagata, and Y. Yamazaki, Phys. Rev. Lett. 97, 213001 (2006).

[17] F. Robicheaux, Phys. Rev. A 73, 033401 (2006).

[18] T. M. Squires, P. Yesley, and G. Gabrielse, Phys. Rev. Lett. 86, 5266 (2001).

[19] T. Northrop, The Adiabatic Motion of Charged Particles (Wiley, New York, 1963).

[20] R. G. Littlejohn, Phys. Fluids 24, 1730 (1981).

[21] G. Gabrielse, Adv. At. Mol. Opt. Phys. 45, 1 (2001).

[22] G. Gabrielse, W. S. Kolthammer, R. McConnell, P. Richerme, R. Kalra, E. Novitski, D. Grzonka, W. Oelert, T. Sefzick, M. Zielinski, D. Fitzakerley, M. C. George, E. A. Hessels, C. H. Storry, M. Weel, A. Müllers, and J. Walz (ATRAP Collaboration), Phys. Rev. Lett. 106, 073002 (2011).

[23] M. E. Glinsky, T. M. O’Neil, M. N. Rosenbluth, K. Tsurula, and S. Ichimaru, Phys. Fluids B 4, 1156 (1992).

[24] G. Gabrielse et al., Phys. Lett. B 507, 1 (2001).

[25] J. L. Hurt, P. T. Carpenter, C. L. Taylor, and F. Robicheaux, J. Phys. B 41, 165206 (2008). 